\title{
Phospholipase A2, Membrane Associated
}

National Cancer Institute

\section{Source}

National Cancer Institute. Phospholipase A2, Membrane Associated. NCI Thesaurus. Code C34063.

Phospholipase A2, membrane associated (144 aa, 16 kDa) is encoded by the human PLA2G2A gene. This protein plays a role in the metabolism of phospholipids. 